\title{
Emociones y Taller de escritura en adultos mayores sobrevivientes del Holocausto
}

Diego Borisonik ${ }^{2}$

\section{Resumen}

La presente investigación tiene como objetivo evaluar y describir el tipo de emociones que surgen en el taller de escritura dictado en la Fundación Tzedaká, en adultos mayores de 73 años sobrevivientes del Holocausto, residentes en la Ciudad Autónoma de Buenos Aires. Para ello, los objetivos se han dividido en tres. Primero, describir las emociones que los participantes manifiestan experimentar antes, durante y después de escribir el relato; y al momento de compartirlo. Segundo, describir las emociones observadas en el momento en que se comparte el relato con el grupo que acude al taller. Y por ultimo, analizar las emociones que aparecen en el material escrito.

Para llevar a cabo el presente estudio de tipo descriptivo se ha entrevistado a siete participantes, un hombre y seis mujeres, de nacionalidad polaca, rusa, francesa, lituana y alemana, de entre 73 y 92 años. Asimismo, se ha llevado a cabo la observación no participante en el taller de cada sujeto seleccionado; y el análisis de las emociones que surgen del escrito.-

El análisis de los datos obtenidos han demostrado que escribir y compartir el relato, sea cual fuere la temática y género, es beneficioso, generando emociones positivas en este grupo de personas de tercera edad, y más precisamente, sobrevivientes de situaciones altamente traumáticas.

Palabras clave: Escritura, Emociones Positivas, Tercera Edad.

Recibido 14-2-2012 | Aprobado 27-4-2012

Institución. Facultad de Ciencias Sociales. Universidad de Palermo

boris_diego@hotmail.com

2 Lic. en Psicología. Investigador Asistente, Universidad de Palermo. 


\section{Writing Workshop and Emotions in senior Holocaust survivors}

\section{Abstract}

The goal of this research is to evaluate and describe the kind of emotions that emerge in senior Holocaust survivors over 73 years old at the writing workshop held at Tzedaka Foundation. For this purpose, the goals have been divided into three. Firstly, the description of the emotions the participants claim to feel before, during and after performing their writing task, and at the moment of sharing it with their peers. Secondly, the description of the observed emotions of the participants at the moment of sharing their writing task with their workshop peers. Lastly, the description of the emotions that emerge from the writing itself.

In order to carry out this descriptive study, seven Holocaust survivors living in the City of Buenos Aires have been interviewed: one man and six women of Polish, Russian, French, Lithuanian and German nationalities, ranging from 73 to 92 years of age, all part of the above mentioned writing workshop. By the same token, the detached observation of each subject in the workshop and the analysis of the emotions arising from the text have been carried out.

The analysis of the resulting data has shown that writing and sharing their writing tasks, whatever the topic or genre, is beneficial and it generates positive emotions in this senior adults' group, and more specifically, highly traumatic situations' survivors.

Key words: Writing, Positive emotions, Senior adults. 


\section{Emociones y taller de escritura en adultos mayores sobrevivientes del Holocausto}

Para poder describir el tipo de emociones y su relación con la escritura, es preciso en primer lugar, brindar una definición de las variables y constructos fundamentales aquí evaluados.

Según Fernández-Abascal, Díaz y Domínguez Sánchez (2001) la emoción es un proceso psicológico adaptativo, que recluta y coordina el resto de los procesos psicológicos cuando las condiciones de una situación exigen una respuesta rápida y efectiva para afrontar y ajustarse a las exigencias y cambios ambientales. Por ello, diríamos que las emociones alteran o modifican el resto de los procesos psicológicos, cargando de afecto la percepción, activando la memoria, movilizando cambios fisiológicos, dirigiendo la atención, planificando acciones, la comunicación verbal y no verbal, motivando a la acción, etc.

Según Castro Solano (2010), las emociones tienen un objeto (un estímulo determinado), son inestables, transitorias, momentáneas y puntuales. Permiten la supervivencia y adaptación al medio, y en el caso de las emociones positivas, si logran mantenerse a lo largo del tiempo, son generadoras de recursos personales, psicológicos, físicos y sociales. De hecho, una persona que logra mantener niveles adecuados de emociones positivas será más resiliente frente a situaciones adversas que otra que no lo logra.

Según diferentes teorías sobre las emociones (La Tradición Darwiniana o Teoría de las emociones básicas; La teoría del Appraisal o Perspectiva Cognitiva y el Constructivismo o Construccionismo Social ) es que se ha ido clasificando a las mismas en primarias y secundarias; negativas, positivas o neutras; desagradables; empáticas; existenciales; pasadas (la gratitud y el perdón), presentes, (el fluir, el saborear y el darse cuenta), futuras (el optimismo y la esperanza), etc. (Castro Solano, 2010).

De entre las clasificaciones, vamos a centrarnos en las emociones que refieren al sentimiento de agrado o desagrado que producen en el sujeto. De allí, surgirían las emociones positivas, negativas y neutras. Las emociones positivas, son aquellas que implican sentimientos agradables, que surgen de la valoración de una situación como beneficiosa, suelen tener una duración temporal muy corta y movilizan escasos recursos para su afrontamiento (Ej. felicidad, humor, serenidad, alivio, simpatía, gratitud, etc.). Las negativas, implican sentimientos desagradables, surgen de la valoración de una situación como dañina y movilizan muchos recursos para su afrontamiento (Ej. ira, miedo, asco). Por último, están las neutras (Ej. la sorpresa) que, caracterizadas por no producir intrínsecamente reacciones agradables ni desagradables, tienen como finalidad el facilitar la aparición de otras emociones (Fernández-Abascal, Díaz \& Domínguez Sánchez, 2001). 
Fredrickson (2000), mediante su Modelo de Ampliación y Construcción de Emociones Positivas, ha llegado a la conclusión que emociones positivas amplían las tendencias de pensamiento y acción, provocando el efecto contrario a las emociones negativas. A partir de esta ampliación se favorece la construcción de recursos personales para afrontar las adversidades (ayudando a mantener los esfuerzos perseverantes para oponerse a las dificultades). Y es esta construcción de recursos personales la que transforma a la persona, tornándola más creativa, flexible, resistente a las dificultades y socialmente mas integrada.

Ahora bien, el enfoque del ciclo vital, entiende que la vejez es la mas heterogénea de todas las etapas vitales ya que se produce en ella una acumulación de diferencias que se han desarrollado a lo largo de toda la vida, tanto normativas respecto de la edad (biológicas y sociales), como de la historia sociocultural del ambiente en que se ha vivido y de las características especificas de la vida de cada persona (Conde Sala, 2007).

Antecedentes en investigación arrojan resultados contradictorios comparando afectividad en adultos jóvenes y adultos mayores. Gatz, Kals-Godley y Karel (1996) hallaron mayores niveles de tristeza en adultos mayores que en jóvenes, pero menores niveles de ira. Charles, Cartensen (2007) y Gross (1998) encontraron que la intensidad en la experiencia emocional es similar en adultos mayores y adultos jóvenes, mientras que la frecuencia de emociones negativas en los primeros es menor. Los mayores experimentarían una reducción en la frecuencia de estas últimas emociones a partir de los 60 años, e igualarían dicha frecuencia a los más jóvenes en lo que hace a las experiencias positivas (Cartensen et al., 2000).

Estudios realizados en España señalan que los adultos mayores y jóvenes experimentan el mismo nivel de afectividad positiva y negativa (Márquez González, Fernández de Trocóniz, Montorio Cerrato \& Losada Baltar, 2008), mientras que en Alemania se han encontrado diferencias en algunos afectos puntuales, como jovialidad, serenidad, sorpresa y tristeza, en los que los adultos mayores puntuaron mas alto (Gruhn, Kotter-Gruhn \& Rocke, 2010).

Lo cierto es que todas las diferencias que se han ido dando entre los distintos estudios e investigaciones, pueden deberse a los aspectos históricos y socioculturales que influyen en cada grupo en particular (Giuliani \& Arias 2010).

Un estudio realizado en Argentina, en la ciudad de Mar del Plata de la Provincia de Buenos Aires en 2010, arrojó que los adultos mayores poseen niveles de afectividad positiva y negativa muy similares a los de los adultos jóvenes. Por lo que ello aportaría una evidencia en contra de los modelos teóricos que afirman la pérdida y deterioro en la vejez como hecho generalizado (Giuliani \& Arias 2010).

Cartensen (1993) realizó una serie de estudios sobre emoción positiva y negativa en amplias muestras de distintas edades, llegando a la conclusión 
de que las personas mayores experimentan igual cantidad de sentimientos positivos que los más jóvenes, y que reducen los sentimientos negativos. Es decir, según las investigaciones realizadas por la autora, en la vejez existiría mayor diferenciación, complejidad, balance y control emocional (Teoría de la Selectividad Socioemocional SST). La cantidad de experiencia acumulada por las personas mayores a lo largo de la vida les proporcionaría mayor capacidad para ejercer control sobre sus emociones y optimizar la experiencia emocional, evitando una sobrecarga.

Otra de las teorías que ofrece claves para entender el bienestar en la vejez es la esbozada por Fredrikson (2001), broaden-and-build theory, acerca de las emociones positivas. Según esta teoría los afectos positivos son un potenciador de recursos psicológicos y comportamentales positivos que alcanzarían su punto cúlmine en la vejez, permitiendo esto un envejecimiento óptimo (Fernández Ballesteros, 2008).

Con ello, Vázquez, Hernangomez y Hervás (2003) ponen de manifiesto que las emociones positivas no solo son generadoras y protectoras de recursos personales necesarios para afrontar el presente, sino que prolongan la vida. Esto se ha visto reflejado a partir de los resultados obtenidos en el llamado Estudio de las Monjas, realizado por Danner, Snowdon, y Friesen, (2001).

Según Pressman y Cohen (2005), quienes han revisado la literatura científica acerca de la asociación entre afecto positivo y condiciones biomédicas (longevidad, mortalidad, supervivencia, recuperación de enfermedades, etc.), la mayor parte de los estudios esta basado en adultos mayores, donde el afecto positivo sería el denominador común como factor protector de la salud mental. En dicha bibliografía han encontrado que las emociones positivas están asociadas a una mayor y mejor longevidad, por lo que potenciarlas mediante actividades placenteras, en frecuencia e intensidad, a lo largo de la vida y en la vejez misma, permitiría envejecer de manera más óptima y exitosa.

Lo cierto es que la emoción es motivo de una conducta y a su vez es generada por ella. Una de sus funciones es la comunicativa, por lo que los actos de comunicación son generados por emociones y el comunicar genera éstas. Según Bruner (1997), la escritura, es una de las diferentes modalidades de expresión narrativa. La narrativa, en general (oral y escrita), es el centro de la construcción de la experiencia humana. Los eventos que componen la narrativa forman parte de las creencias y los deseos de las personas que narran. El narrar una historia no se constituye en un acto que pueda considerarse como verdadero, sino como la posibilidad de llegar a serlo, guardando cierta verosimilitud dentro de la experiencia vivida por el sujeto.

Según Freeman, Epston y Lobovits (2001), la trama de los relatos organiza la información que procede del flujo de las experiencias vividas y convierte algunos 
momentos en un relato significativo sobre los acontecimientos. Así, los relatos o escritos que se organizan con percepciones o emociones negativas tienden a convertirse en relatos dominados por el problema, destacando en él experiencias negativas y las atribuciones negativas de la motivación, las intenciones y el carácter. Mientras que los relatos de esperanza y experiencias positivas tienden a basarse en características e incidentes de la vida de la persona que están al margen del problema.

Siguiendo a Bruder (2011), los seres humanos cuentan con un potencial único para crear e imaginar, lo que puede verse reflejado en la escritura como una de las formas de su expresión. Es a través de ella, que el creador de un cuento puede tomar una distancia óptima si se trata, por ejemplo, de escribir acerca de un trauma o sufrimiento intenso permitiendo ello manipular y desarticular mejor el conflicto, utilizándolo como objeto de reflexión.

Una de las características más importantes de la escritura, sino la más importante, es la manera en que facilita la expresión de las emociones, es su gran poder catártico. Cuando se escribe se libera lo que se lleva dentro. Existe un desbloqueo emocional intenso, en el que el pensamiento, la emoción y la palabra escrita se comprometen en el escrito. De allí, es que a través de la escritura las personas que atraviesan situaciones de estrés mejoran su bienestar psicológico y físico (Pennebaker, 1997).

La escritura anónima en primera persona ha ido tomando valor científico gracias a numerosas investigaciones que le han dado a dicha herramienta de expresión un valor notable en beneficio de la salud psicofísica (Bruder, 2011). Según el estudio realizado por Bruder en el año 2002, escribir un cuento en tercera persona, acerca de la situación más dolorosa vivida en el pasado, concluyéndolo con un final positivo, mejora el bienestar psicológico, aumenta las emociones positivas, disminuyendo la afectividad negativa y la sintomatología física (Bruder, 2000, 2004).

De acuerdo con un estudio realizado por Pennebaker en la década del 80, se ha demostrado que las personas que escribieron acerca de sus pensamientos, emociones y sentimientos más profundos relacionados con un trauma, mejoraron los niveles de salud, reduciéndose a un 50\% el número de visitas a los médicos. Mejoraron la actividad inmunológica, disminuyendo su tensión y malestar físico, demostrando ser aquella, una herramienta que alivia experiencias emocionales negativas (Pennebaker \& Segal, 1999).

Un estudio llevado a cabo por Nye (1997) reveló que la escritura mejora la salud física y mental de pacientes con HIV. En esta investigación la escritura no fue anónima, más bien se escribió en forma privada y luego se leyó en grupo lo producido. En este proceso de escribir y compartir se notó una mejora a nivel psicofísico importante en los pacientes enfermos. 
Una investigación que relacionaba la escritura con afectos positivos y negativos, arrojó que las personas con mayor sintomatología física, invitados a escribir sobre su situación traumática, fueron los que describieron mayores emociones positivas, mientras que aquellas personas que presentaban mayor estado de salud física (es decir, que habían mejorado) describían mayormente emociones negativas en sus escritos. Esto llevó a concluir que expresar las emociones negativas (dolor, desilusión, bronca, culpa, etc.) produce en el sujeto un efecto catártico que le permite mejorar su salud (De Salvo, 1999).

King (2001) en una variante del paradigma de escritura expuesta por Pennebaker, realizó un estudio y obtuvo, que escribir acerca de sucesos emocionales positivos tiene los mismos beneficios para la salud, que el hacerlo sobre sucesos traumáticos, ya que los grupos evaluados arrojaron iguales niveles de bienestar subjetivo.

Concluyendo, Pennebaker (1999) sostiene que escribir acerca de situaciones traumáticas vividas por la persona puede volverlo mas saludable, destacando así el valor terapéutico de la escritura. El escribir de manera libre sobre pensamientos, emociones y acciones permite la relajación, promueve la salud física y mental, mejorando la calidad de vida.

En virtud de los antecedentes teóricos y empíricos, se determinaron los siguientes objetivos:

1. Describir las emociones que los participantes manifiestan experimentar antes, durante y después de escribir el relato; y al momento de compartirlo.

2. Describir las emociones observadas por el pasante en el momento que se comparte el relato con el grupo que acude al taller.

3. Analizar las emociones que aparecen en el material escrito.

\section{Método}

\section{Participantes}

Se trabajó con participantes que asistían al Taller del Escritura de la Fundación Tzedaká, residentes en de la Ciudad Autónoma de Buenos Aires.

La unidad de análisis lo fueron siete participantes sobrevivientes del Holocausto, un hombre y seis mujeres, de nacionalidad polaca, rusa, francesa, lituana y alemana, de entre 73 y 92 años, que formaban parte del taller mencionado.

Estos fueron sugeridos y autorizados por la Fundación, de acuerdo al estado de salud psíquico-emocional para colaborar con el presente trabajo.- 
Tres de los participantes contaban con estudios secundarios completos, dos de ellos solo primarios y los dos restantes Universitarios. La mayoría había ingresado al taller hace ocho años (desde la creación), y solo dos de los participantes lo habían hecho más recientemente, hacía dos años.-

\section{Instrumentos}

1. Entrevista Semiestructurada: La misma fue orientada con el fin de obtener información acerca de las emociones que siente el sujeto antes de sentarse a escribir, durante el acto de escritura, inmediatamente a posteriori y al momento de compartir su relato en el taller. Se intentó obtener la información precisa acerca de cuáles son las emociones que manifiesta haber tenido el sujeto en los distintos momentos de producción y exposición. Qué sintió corporalmente, qué pensó, cuándo y a partir de qué le surge la emoción, etc. Se diseñó una entrevista ad-hoc para los propósitos del presente estudio.

2. Observación: Se llevó a cabo la observación no participante en el taller de escritura de cada sujeto seleccionado, al momento de exponer y compartir su producción. Se tomó un registro escrito de las emociones que se observaron en el participante expositor. Se observó la postura que tenía al leer, su gesticulación, el tono de voz, los comentarios que hacia al leer, etc. Se diseño una grilla ad-hoc para los propósitos del presente estudio.

3. Relatos escritos: Se llevó a cabo, mediante lectura y análisis, la descripción de las emociones que aparecen en el cuento/ escrito producido y luego compartido.

El criterio para evaluar y analizar los tipos de emociones presentes en el relato fueron determinando el tipo y cantidad de palabras positivas y negativas que poseyó el relato; qué ocurrió con el personaje de aquel; cómo comienza, se desarrolla y culmina la historia; sobre qué temática se ha escrito; en qué persona esta narrado el cuento (distanciamiento); puntuación; etc. Se diseño una grilla adhoc para los propósitos del presente estudio.

\section{Procedimiento}

Se seleccionaron siete sujetos que asisten al taller de escritura para sobrevivientes del Holocausto, dictado en la Fundación Tzedaká, con quienes se acordó un encuentro para realizar una entrevista de carácter semiestructurado. La misma tuvo aproximadamente una duración de 10 minutos. 
La consigna sobre la temática a escribir fue dada con una semana de antelación, y la misma consistió en escribir sobre "Lo Inesperado". Ante ello a los participantes se les solicitó que intenten identificar y tomar registro por escrito de todo sentimiento y emoción que experimentaran antes, durante y al culminar su escrito, así como identificar las emociones que le surgieran al momento de leer y exponer su escrito frente al grupo.

A la semana siguiente, el día de la exposición y al término del taller, se llevó a cabo la entrevista de carácter semiestructurada con cada uno de los participantes en la que se indagó acerca de lo que sintió corporalmente y pensó en cada uno de los momentos (antes, durante, luego y al compartir).

Por otra parte, se procedió a tomar nota de las emociones que pudieron observarse al momento en que el participante compartió su escrito con el resto del grupo en el taller, analizando qué y cómo se expresa. Se observó la postura, gesticulación, entonación y reacción a los comentarios del resto de los participantes y la coordinadora.

Por último, se analizó el tipo de emociones que aparecieron de los propios escritos.

\section{Resultados}

Con el propósito de analizar el tipo de emociones que surgieron en el taller de escritura para adultos mayores, describiendo para ello las emociones expresadas por los participantes, las observadas al momento de compartir su escrito y las surgidas en los textos, se ha llevado a cabo una entrevista de carácter semiestructurado con cada participante seleccionado para que informen acerca de toda emoción que experimentaron al momento previo de sentarse a escribir; cuando se encontraban escribiendo; al culminar su escrito, así como también, al momento de compartirlo con el grupo.

Por otra parte, se procedió a la observación de las emociones expresadas al momento en que el participante compartió su escrito con el resto del grupo en el taller, y por último, se analizó el tipo de emociones que aparecieron de los propios escritos.

Los resultados de ello se ven reflejados en la Tabla 1. 


\section{Tabla 1 I Emociones de los participantes antes, durante, después y al compartir el escrito; las transmitidas por estos durante la lectura en público y las que surgen del texto escrito.}

\begin{tabular}{|c|c|c|c|c|c|c|}
\hline Participantes & Antes & Durante & Después & Al Compartir & Observación & Texto \\
\hline Mujer & $(+)$ & $(+)$ & $(+)$ & $(+)$ & $(+)$ & $(+y-) a(+)$ \\
\hline Mujer & $(+)$ & $(+)$ & $(+)$ & $(+)$ & Neutra & $(+)$ \\
\hline Mujer & $(-)$ y $(+)$ & $(-)$ y $(+)$ & $(+)$ & $(+)$ & $(+)$ & $(+y-)(+)$ \\
\hline Varón & $(-)$ & $(-)$ & $(+)$ & $(+)$ & $(-)$ & $(-) \mathrm{a}(+)$ \\
\hline Mujer & $(-)$ & $(-)$ y $(+)$ & $(+)$ & $(+)$ y $(-)$ & $(-)$ & $(-) \mathrm{a}(+)$ \\
\hline Mujer & $(+)$ & $(+)$ & $(+)$ & $(+)$ & $(+)$ & $(-) \mathrm{a}(+)$ \\
\hline Mujer & $(-)$ & $(-)$ & $(+)$ & $(+)$ y $(-)$ & $(-)$ & $(-)$ \\
\hline
\end{tabular}

Del análisis de los datos recabados, puede decirse que los textos, en su mayoría, han de reflejar emocionalmente lo que ha manifestado sentir cada uno de los participantes durante el proceso de escritura. Si observamos la Tabla 1, las emociones experimentadas en el durante tienden a coincidir y/o aparecer en el texto escrito (véase columna 3 y 7). Puede que del texto surjan otras emociones, mas en ninguno de los casos se han opuesto.

A partir del cuadro se puede inferir que las emociones antes y durante la actividad de escribir tienden a coincidir entre sí, y a su vez coinciden con las que surgen del texto escrito (véase columnas 2,3 y 7). Al menos no se encuentran contrariadas. En ninguno de los casos, en el antes y durante, se experimenta un tipo de emoción y en el texto escrito, surge su opuesto.

En lo que hace a las observaciones de las emociones transmitidas, la mayoría de los participantes han expresado facial y posturalmente el mismo tipo de emociones que han mencionado experimentar al momento de compartir su relato en público (véase columna 5 y 6). Solo en el caso de dos participantes, las emociones no coinciden. En la mayoría de los casos lo sentido (subjetivamente) y expresado (objetivamente) tienden a coincidir (Ekman, 1972, 2003).

De los resultados arrojados por el presente estudio se podría decir que escribir, ya sea sobre aspectos positivos como negativos, llevaría a experimentar emociones positivas en lo que hace a este grupo en particular (véase columna 4). 
Ninguno de los participantes ha experimentado una emoción negativa luego del escribir, aunque su texto haya concluido con un final negativo, lo que coincide con el estudio realizado por King (2001).

Por último, del análisis de los datos obtenidos podemos concluir que al momento de compartir el escrito, todos los participantes han manifestado experimentar emociones positivas, amén de que dos de ellos también hayan experimentado emociones negativas. Por lo que compartir los escritos mejoraría los niveles emocionales (Nye, 1997).

\section{Discusión}

El presente estudio ha descripto las emociones experimentadas y transmitidas por los participantes de tercera edad sobrevivientes del Holocausto, que concurren al taller de escritura dictado en la Fundación Tzedaká.

A partir del presente trabajo se ha podido concluir acerca de la importancia de la escritura como generadora de emociones, y más precisamente de emociones positivas, tanto al concluir un escrito, como al compartirlo con el grupo.

En este caso, sin importar cuándo han comenzado con el taller de escritura, a todos por igual, escribir y compartir sus escritos, les produjo el mismo efecto: emociones positivas.

El valor que tiene el escribir acerca de nuestros pensamientos y sentimientos estriba en la reducción de la inhibición, y en organizar nuestra vida mental y emocional. Ayuda a mantener la brújula psicológica manteniendo en buen estado nuestra salud (Pennebaker, 1999).

Lo cierto es que el análisis de los datos obtenidos coinciden con investigaciones mencionadas a lo largo del presente (King (2001), Bruder (2011), Pennebaker (1997), Nye (1997), etc.) en tanto escribir, mejoraría la actividad inmunológica, disminuiría la tensión y el malestar físico, demostrando ser aquella, una herramienta que alivia experiencias emocionales negativas.

Observando las conclusiones a las que se llega en éste y otros estudios, el taller de escritura, que invita y promueve la escritura, así como el compartir la misma con otros (creando redes sociales) genera emociones positivas, mejorando el bienestar de las personas. Por lo que si escribir y compartir el relato, sea cual fuere la temática y género, es beneficioso, debiera colocarse mayor atención e importancia en esta actividad en lo que hace al trabajo con personas de tercera edad, y más precisamente con sobrevivientes de situaciones altamente traumáticas, es decir, tomar en cuenta la escritura como herramienta beneficiosa para su salud.

De los resultados del presente, surge que un mismo disparador temático ha generado una pluralidad de emociones, mas todas han desembocado en 
positivas, sin importar si se ha escrito sobre situaciones favorables o negativas. Si bien factores como rasgos de personalidad o sucesos propios de la vida por los que transita cada participante, no han sido tomados en cuenta y pueden teñir los resultados obtenidos, lo cierto es que ha sido llamativo que en el $100 \%$ de los casos escribir ha generado bienestar, y ninguno de los participantes ha mencionado considerar dicha actividad como negativa, muy por el contrario. Esto coincidiría con los aspectos desarrollados por Carteasen (1993), en su teoría de la Selectividad Socioemocional.

Los problemas de memoria no inhabilitan, en principio, la fantasía y capacidad creativa de un ser humano, que pueden verse expresadas a través de la escritura. De hecho, esta actividad puede llegar a mejorar ciertas habilidades cognitivas, toda vez que ordena, organiza y permite incorporar nueva información, es facilitadora en el proceso de aprendizaje, posibilita la flexibilidad de pensamiento e imaginación y genera emociones positivas (Pennebaker,1997).

Este estudio, que ha tenido intención de describir emociones emergentes de la escritura, también abre la posibilidad a futuras investigaciones que puedan centrarse en determinar si ésta, sin importar el género y la temática (dramática, descriptiva, informativa, etc.) es generadora de emociones positivas, es decir, si el recurso de la escritura libre mejora la calidad de vida de las personas, constituyéndose como una herramienta indispensable en el área de la Psicología Positiva.-

Por otro lado, podría completarse el presente estudio con uno de tipo longitudinal, realizando un seguimiento en el tiempo de los participantes, a fin de obtener mayor información acerca de la duración de las emociones positivas luego de la escritura y determinar si las emociones positivas experimentadas al culminar el escrito, perduran más en tiempo si la persona ha escrito sobre situaciones traumáticas ( $\mathrm{y}$ ha experimentado antes y durante el proceso de escritura emociones negativas) que si lo ha hecho sobre situaciones favorables.

Seria interesante dividir en tres etapas (antes, durante y luego) las emociones que experimenta la persona al momento de compartir su relato con el grupo, ya que lo cierto es que el momento de compartir lo escrito, como acto, también tiene sus tres tiempos que no han sido aquí reflejados y diferenciados.

Asimismo, futuras investigaciones podrían realizarse longitudinalmente, haciendo un seguimiento en meses y años de los distintos momentos en que la persona escribe (antes, durante, luego y al compartir el texto), y sobre distintas temáticas, para obtener con mayor precisión resultados acerca de las emociones que genera la escritura, ya que en el presente, la descripción de las emociones se ven acotadas a un solo y único disparador temático, pudiendo existir innumerables variables que modifiquen las conclusiones a las que se llega en el presente: tiempo, temática, entrenamiento, estado de ánimo, etc. 
Otra posibilidad podría consistir en evaluar las emociones dividiendo en grupos a los participantes en función del tiempo que hace que concurren al taller, para evaluar si existen diferencias en el control de las emociones de acuerdo a la experiencia y tiempo de asistencia al taller.

Por último, interesante sería llevar a cabo estudios correlacionales de diferencias de grupos para investigar acerca de los efectos de la escritura en otros grupos etáreos, que hayan y no, sobrevivido a una guerra, y luego compararlos entre sí, para analizar las diferencias y el impacto que genera dicho recurso en las emociones a distintas edades.

Para concluir, es destacable admitir que el análisis de los datos en el presente estudio deja de manifiesto que escribir generaría emociones positivas, las que permitirían ampliar el repertorio de pensamiento-acción, construyendo y/o reforzando recursos personales (intelectuales, físicos y psicosociales). Si ponemos en juego y reforzamos dichos recursos a lo largo de la vida, y también durante la vejez, se obtendrían mayores habilidades adaptativas (incluyendo el manejo de situaciones estresantes). En conclusión, los afectos positivos son un potenciador de recursos psicológicos y comportamentales positivos que alcanzarían máxima expresión en la vejez, permitiendo esto un envejecimiento óptimo (Fernández Ballesteros, 2008). Es decir, para llegar a una vejez óptima y lo más sana posible, es necesario aumentar la calidad de nuestros momentos presentes vividos, ya que serán la base del recuerdo futuro. Por lo que, si mediante un recurso como la escritura lográramos generar emociones positivas, el promover dicha práctica nos beneficiaría psico-físicamente no solo en el presente, sino también, a futuro.

\section{Referencias bibliográficas}

Bruder, M. (2000). El cuento y los afectos. Buenos Aires: Galerna.

Bruder, M. (2004). Implicancias del cuento terapéutico en el bienestar psicológico y sus correlatos. Tesis doctoral. Universidad de Palermo. Buenos Aires. Argentina.

Bruder, M. (2011). Escritura y cuento terapéutico. Buenos Aires: Hormé.

Bruner, J. (1997). La Educación puerta de la cultura. Madrid: Aprendizaje Visor. 
Castro Solano, A. (2010). Las Emociones Positivas: Fundamentos de la Psicología Positiva. Buenos Aires: Paidós.

Carstensen, L.L. (1993). Motivation for social contact across the life span: A theory of socioemotional selectivity. En J.E. Jacobs (Ed.): Nebraska Symposium on Motivation. Developmental Perspectives on Motivation. Vol. 40 (pp. 209-254). Lincoln: University of Nebraska Press.

Cartensen, L.L., Pasupathi, M., Mayr, U. y Nesselroade, J. (2000). Emotional Experience in everyday life across the adult life span. Journal of Personality and Social Psychology, 79, 644-655.

Charles, S.T., Mather, M. y Cartensen, L. L. (2007). Emotion regulation and aging. En J.J. Gross (Ed). Handbook of Emotion Regulation. Nueva York: Guilford Press.

Conde Sala, J. (2007). Psicogerontología postitiva: Cambios en las concepciones. Inter- psiquis 2007 (on line). Disponible en www.psiquiatria.com. Crawford, J. \& Henry, J. (2004). The Positive and negative affect Schedule (PANAS): Construct validity, measurement properties and normative data in a large non-clinical simple. British Journal of clinical psychology, 43,245-265.

Danner, D.D., Snowdon, D.A. \& Friesen, W.V (2001). Positive emotions in early life and longevity. Findings from the nun study. Journal of Personality and Social Psychology, 80(5),804-813.

De Salvo, L. (1999). Writing as a Way of Healing. Harper San Francisco. New York: A Division of Harpers Collins Publishers.

Ekman, P. (2003). Emotions revealed. Recognizing faces and feeling to improve communication and emotional life. Nueva York: Henry Holt.

Ekman, P. (1972). Universals and cultural differences in facial expressions of emotion. Nebraska symposium on motivation. Nebraska: University Of Nebraska Press.

Freeman, J., Epston, D. \& Lobovits, D. (2001). Terapia narrativa para niños. Barcelona: Paidós. 
Fernández-Abascal, M. Martín Díaz \& J. Domínguez Sánchez (2001). Procesos Psicológicos. Madrid: Pirámide.

Fernández Ballesteros, R. (2008). El bienestar en los mayores. En C. Vázquez Valverde y G. Hervás Torres (Eds.) Psicología positiva aplicada. Madrid: Desclée de Brouwer. (pp. 371-399).

Fredrickson, B. L. (2000). Positive emotions. En C. R. Snyder y S. J. López (Eds.) Handbook of Positive Psychology. New York: Oxford University Press. (pp. 120-134).

Fredrickson, B. L. (2001). The role of positive emotions in positive psychology: The broaden -and-build theory of positive emotions. American Psychologist, 56, 218-226.

Gazt M., Kals-Godley, J. \& Karel M. (1996). Aging and mental disorders. En Birren, J. \& Schiane (Eds.). Handbook of psychology of aging. San Diego: Academic Press en Charles, S.T., Mather, M. y Cartensen, L. L. (2007). Emotion regulation and aging. En J.J. Gross (Ed). Handbook of Emotion Regulation. Nueva York: Guilford Press.

Giuliani, M. F. \& Arias, C. J (2010) La afectividad en adultos jóvenes y adultos mayores de la ciudad de Mar del Plata: un estudio comparativo. II Congreso Internacional de Investigación y Práctica Profesional en Psicología, XVII Jornadas de Investigación y Sexto Encuentro de Investigadores en Psicología del MERCOSUR. Buenos Aires. V. 3, 91-93.

Gross, J.J. (1998). Antecedent-and response-focused emotion regulation: Divergent consequences for experience, expression, and psychology. Journal of personality and social psychology, 74, 224-237.

Gruhn, D, Kotter-Gruhn, D. \& Rocke, C. (2010). Discrete affects across the adult life span: Evidence for multidimensionality and multidirectionality of affective experiences in young, middle-aged and older adults. Journal of research in personality. En prensa.

King, L. (2001). The health benefits of writing about life goals. Personality and Social Psychology Bulletin, 27, 798-807. 
Márquez González, M., Fernández de Trocniz, M., Montorio Cerrato, I. \& Losada Baltar (2008). Experiencia y regulación emocional a lo largo de la etapa adulta del ciclo vital: análisis comparativo en tres grupo de edad. Psicothema, 20 (4), 616-622.

Nye, E. F. (1997). Writing as healing. (writing improves mental health of HIV patients). Qualitative inquiry. 3 (4) 439-452.

Pennebaker, J. W. (1997). El arte de confiar en los demás. Alianza: Buenos Aires.

Pennebaker, J. W. \& Segal J. D. (1999). Forming a Story: The health benefits of narrative. Journal of Clinical Psychology, 5, 1243-1254.

Pressman, S.D. \& Cohen S., S. (2005). Does positive affect influence health? Psychological Bulletin, 131, 325-971.

Vázquez, C., Hernangomez, L. \& Hervás, G. (2003). Longevidad y emociones positivas. En L. Salvador, A. Con, J.R. Cabo y F. Alonso (Dir.): Longevidad y vida saludable. Madrid: AECES. 